\title{
Can low frequency accelerometry replace pressure measurements for monitoring gas-solid fluidized beds?
}

\author{
Lilian de Martín a , Javier Villa Briongos ${ }^{\mathrm{b}, *}$, José M. Aragón a ${ }^{\mathrm{a}}$ María C. Palancar ${ }^{\mathrm{a}}$ \\ ${ }^{a}$ Faculty of Chemistry, Department of Chemical Engineering, University Complutense of Madrid, Av. Complutense s/n, 28040 Madrid, Spain \\ ${ }^{\mathrm{b}}$ Higher Technical School, Department of Thermal and Fluid Engineering, University Carlos III of Madrid, Av. Universidad 30, 28911, Leganés, Madrid, Spain
}

\begin{abstract}
A B S T R A C T
This paper is addressed to introduce a methodology based on low frequency accelerometry that can be used for gas solid fluidized bed monitoring, and for dynamic diagnosis purposes. The proposed methodology consists on extracting the low frequency information encoded within an accelerometry signal, by means of the Hilbert transform method. The time and the frequency domain analysis show how this low frequency information is directly related to the conventional pressure fluctuation measurements, providing useful information on bulk and bubble dynamics. The cross correlation and the coherence function analysis between the pressure and the envelope process of the measured accelerometer signals "E", exhibit values approaching unity for frequencies ranging between 2 and $4 \mathrm{~Hz}$. This reveals that pressure signals and accelerometry envelope are related processes. The results from the Coherent Output Power, COP, and the Incoherent Output Power, IOP, analysis confirms that both pressure and envelope time series exhibit the same global and local features open the possibility of using low frequency accelerometers instead of conventional pressure transducers for monitoring and for dynamic diagnosis purposes.
\end{abstract}

\section{Introduction}

The monitoring of gas solid fluidized beds (FB) has been an important issue since they were first used in industry. As the dynamical behaviour characterizing those systems is complex, it is necessary to monitor different bed properties continuously. As an example of that dynamical complexity, it has been shown that, depending on the operational condition, FB can exhibit dynamical chaotic features (Daw et al., 1990; Schouten and van den Bleek, 1992), which can be used, for instance, to predict malfunctioning (van Ommen et al., 2000, 2004).

During the past, a large research effort has been directed towards developing measurement techniques aimed to get knowledge on the dynamic phenomena that take place within the bed (Werther, 1999; Yates and Simons, 1994); some examples are the capacity and the inductance measurements (Brereton and Grace, 1993; Louge and Opie, 1990), techniques based on optic fiber probes (Amos et al., 1996; Zhang et al., 1998), and laser based measurements (Briongos and Guardiola, 2003; Solimene et al., 2007). However, among those techniques, the pressure fluctuations measurements remain as the most used technique (Johnsson et al., 2000; Sasic et al., 2007). The success of the

\footnotetext{
* Corresponding author.

E-mail address: jvilla@ing.uc3m.es (J. Villa Briongos).
}

pressure monitoring is explained by its low cost and the direct relation that exists between the property measured and the bed dynamics. Moreover, from pressure measurements sampled at relatively low frequency it is possible to extract useful informa tion about the bulk, bubble and wave dynamics (van der Schaaf et al., 2002). Therefore, both the quality and the quantity of the low frequency information contained in the pressure signal make this technique the most used to monitor the bed dynamics. Nevertheless, it has several great disadvantages that compromise its applicability to industrial installations: on one hand, the measurement of pressure fluctuation is a local technique, thus it has been reported that the dynamic measured can be affected by different factors such as the pressure probe itself or by the probe length (van Ommen et al., 1999), moreover, some other additional measurement problems might appear such as solid probe blockage. On the other hand, invasive features should be considered, since it is necessary to perforate the vessel in order to introduce the pressure probes inside the bed, limiting applications under severe, corrosive and high pressure/tempera ture conditions, and pressure probes can represent an obstacle for the free development of bubbles and solid motion.

According to that, the need for non invasive measurement methods is clear, which in principle will offer several advantages with respect to the invasive methods. Thus, because they are located outside the vessel, they neither suffer the harsh operating conditions inside the bed nor do they interfere with the bed 
dynamics. Nevertheless, the application of these methods in industrial fluidized beds is still an unresolved matter; some factors such as the large size of industrial facilities becomes a big challenge for reported non invasive measurement techniques such as tomography, ECT.

During the last decade, the need for non invasive techniques led to the development of acoustic monitoring methods, which are based on microphone/hydrophone and accelerometer mea surements (Boyd and Varley, 2001). Those acoustic techniques are later applied as active or as passive methods (Cents et al., 2004; Herrera et al., 2002; Boyd and Varley, 2002). With regard to the passive acoustic emission in which the signal collected is created by the fluidizing process itself, acoustics measurements have been reported by either placing the sensor inside (Zukowski, 2001) or outside the vessel (Briongos et al., 2006b; Cody et al., 1996; Finney et al., 1998). In spite of the initial advantages of the external acoustic measurements (non invasive and low imple mentation cost), they present some problems. Thus, the measured signal is very complex, and it can be strongly influenced by factors such as the background noise, secondary mechanical vibrations and the vessel response. Moreover, working at ultrasonic measurement ranges is frequently used to avoid noise. Never theless, this would lead to high computational costs due to the great number of data in each sample. For this reason, most of the published papers have used data analysis based on averaged properties, which have been mainly attributed to particle dynamics (Cody et al., 1996; Tsujimoto et al., 2000).

To the best of the authors' knowledge, there are few works in literature that use advanced techniques over external passive acoustic signals for getting low frequency information (Briongos et al., 2006b; Finney et al., 1998). Besides, those previous works deal with the unwanted slugging regime. Consequently, the challenge of finding a low cost, non invasive methodology that can replace the conventional pressure measurements is still open.

This paper is addressed to introduce a methodology based on low frequency accelerometry that fulfils the challenge. The proposed methodology consists on extracting the low frequency information encoded within an accelerometry signal, by analyzing the envelope process of the measured accelerometer signal. This low frequency information is shown to be directly related with the conventional pressure fluctuation measurements and consequently, it provides useful information on bulk and bubble dynamics that can be used for monitoring and dynamic diagnosis purposes.

\section{Accelerometry fundamentals}

The mechanical vibration can be seen as an oscillation motion about a reference position. Moreover, it is well known that the oscillatory motion can originate waves and viceversa. In that way, the vibration measurement has information on the physical phenomena behind the oscillating motion. Thus, in order to understand how the information is encoded, let us consider as first approximation the simpler case, where a physical phenom ena originates a wave that leads to a periodic oscillating motion, in that case the movement can be described by the harmonic motion as sinusoids. Moreover, the wave theory also establishes that different waves can couple to generate a phenomenon known as beats where the information of forming waves is encoded.

In order to characterize the mechanical vibration, it is necessary to measure such an oscillatory motion where both the amplitude and frequency information are encoded. In principle, it should not matter whether the displacement, velocity or accel eration parameters are measured when studying mechanical vibration, since all three quantities are related by integration or differentiation. However, the physical nature of vibrations sources

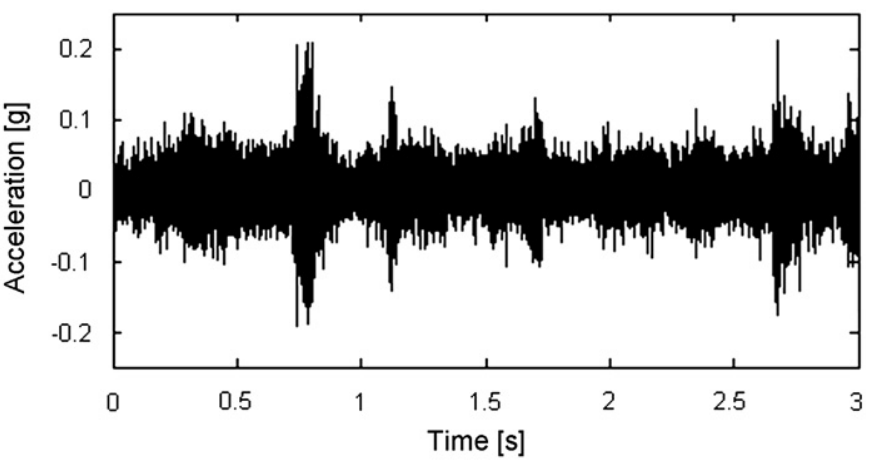

Fig. 1. Experimental accelerometry signal in a FB.

and the characteristics of the transducers used for measuring make one parameter preferred over the others. Thus, it is well known that displacement measurements are suitable when a wide band signal is expected and the low frequency component has to be studied, whereas acceleration, which gives most weight to high frequency components is applied for high frequency studies (Gatti and Ferrari, 1999). However, the accelerometers are most commonly used in practice because of their versatility, small dimensions, wide frequency and dynamic operational ranges, availability and low price. Up to now, the limiting parameter for applying accelerometry to study low frequency phenomena has been the unfavourable signal to noise ratio that characterizes the low frequencies components of the conventional measured accelerometer signals, which used to have poor low frequency response characteristics.

In contrast to conventional accelerometers, in this work, the mechanical vibrations of a FB, are measured with accelerometers with high sensitivity ( $\sim 995 \mathrm{mV} / \mathrm{g}$ ), which allow to study the low frequency information encoded within the measured signal. An example of the measured accelerometry signal is shown in Fig. 1, where the beat phenomena characterizing narrow band signals can be observed.

As stated above, it is well known that, in order to extract the low frequency information of the phenomena behind the measured signal, it is not suitable to use the direct accelerometry signal (Harris and Piersol, 2002). According to that, for extracting the low frequency information, it is a common practice to integrate the power of the signal with a moving root mean square, rms (Norton, 1989). That operation returns the envelope process associated to the signal. Consequently, similar informa tion can be obtained with other techniques such as the Hilbert transform method or the use of nonlinear energy operators (Huang et al., 2009).

Since most of the dynamical phenomena characterizing FB dynamics such as bubble, and bulk dynamics take place at relatively low frequency (generally below $20 \mathrm{~Hz}$ ), this paper focuses on the low frequency information stored within the acceleration signals by using the Hilbert transform method to extract the low frequency information. Furthermore, the time and frequency domain analysis are applied to compare the similarity between the pressure and the envelope acceleration, "E", signals recovered through the Hilbert transform methodology,

\section{Analysis methods}

\subsection{Frequency domain}

The frequency domain analysis has been widely applied to characterize fluidization regimes (Satija and Fan, 1985; Zijerveld 
et al., 1998) and to gain knowledge on the physical phenomena that take place inside a fluidized bed (Kage et al., 1991; Sun et al., 1994). Among the different spectral properties, the power spectrum density (PSD) provides a picture of the characteristic frequencies appearing within a signal. There are different methods to estimate the spectra; here, Welch's method is used for the PSD estimation by averaging 29 periodograms, achieving a good trade off between resolution and error (Brown and Brue, 2001).

It has been reported in literature that pressure fluctuations within the bed can be due to local and global contributions (Bi et al., 1995; Sasic et al., 2007; Bi, 2007). That fact leads to the use of different techniques focused on identifying and distin guishing those contributions from the measured signal. Thus, the multi scale nature of fluidized bed dynamics has drawn con siderable attention in the last years (Li and Kwauk, 2001; Lu and Li, 1999; Zhao and Yang, 2003; Briongos et al., 2006a, 2007; Abbasi et al., 2009). However, due that the causes and sources of accelerometer signals are not yet understood, the Coherent Output Power, COP, and the Incoherent Output Power, IOP, analysis used in van der Schaaf et al. (2002) is applied here as a first approach to analyze the different dynamical components appearing in the envelope process, comparing the local and global information stored in both pressure and envelope accelerometer signals. Consequently, the cross spectral properties measured by the normalized cross PSD function (coherence function), between two or more signals collected at different positions within the bed, have been used to study the joint behaviour of the compared signals, quantifying the linear causality existing between them. Thus, the more correlated the frequencies, the higher the coher ence. According to that, the joint behaviour of plenum and in bed pressure signals is used to perform a preliminary distinction between local and global dynamics from a measured signal.

\subsection{Envelope process and the Hilbert transform}

As stated above, in order to extract the low frequency information contained in the signal measured by the acceler ometers, $a(t)$, the Hilbert transform is used to recover the envelope process, $A(t)$, associated with $a(t)$. As Fig. 2 shows, the envelope process consists of a pair of smoothly varying curves around the measured signal. Considering $a(t)$ as a non stationary dynamical process having evolutionary spectral density, its envelope is given by (Nigam, 1983)

$A(t)=\sqrt{ } a^{2}(t)+b^{2}(t)$,

where $a(t)$ and $b(t)$ are orthogonal non stationary dynamical processes and can be considered as the rectangular coordinates of a point in the $(a, b)$ plane (Fig. 3 ), defined by an analytic signal of the form:

$Y(t)=a(t)+b(t) i$

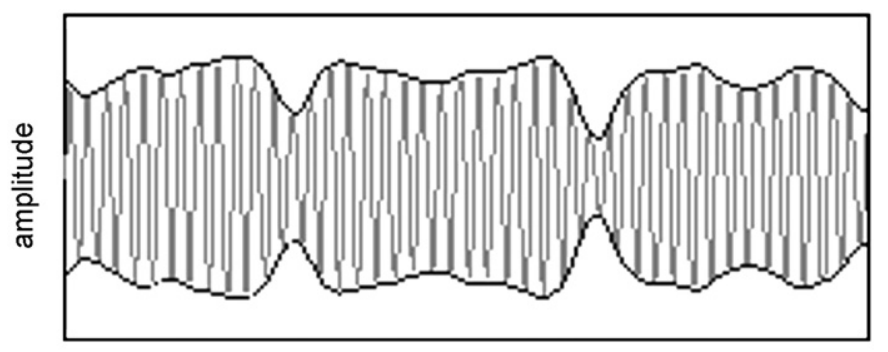

time

Fig. 2. Time series and associated envelopes.

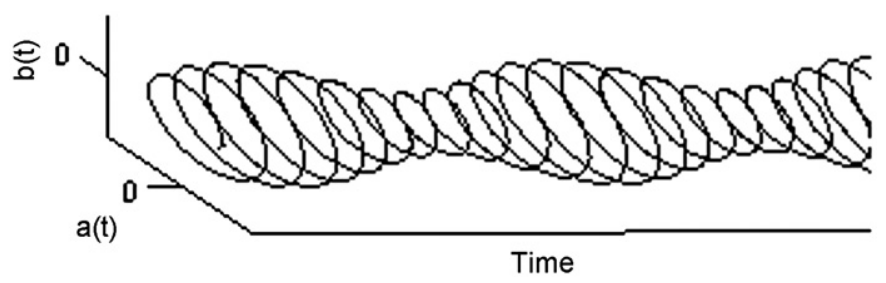

Fig. 3. Temporal evolution of an analytic signal in the complex plane.

Table 1

Fluid-dynamic characteristics of Ballotini glass beads (Group B of the Geldart classification).

\begin{tabular}{llllll}
\hline$d_{\mathrm{p}}(\mathrm{m})$ & $\rho_{\mathrm{mf}}\left(\mathrm{kg} / \mathrm{m}^{3}\right)$ & $\Phi$ & $\varepsilon_{\mathrm{mf}}$ & $\rho_{\mathrm{p}}\left(\mathrm{kg} / \mathrm{m}^{3}\right)$ & $u_{\mathrm{mf}}(\mathrm{m} / \mathrm{s})$ \\
\hline $3.8 \cdot 10^{4}$ & 1462 & 0.9 & 0.407 & 2464 & 0.107 \\
\hline
\end{tabular}

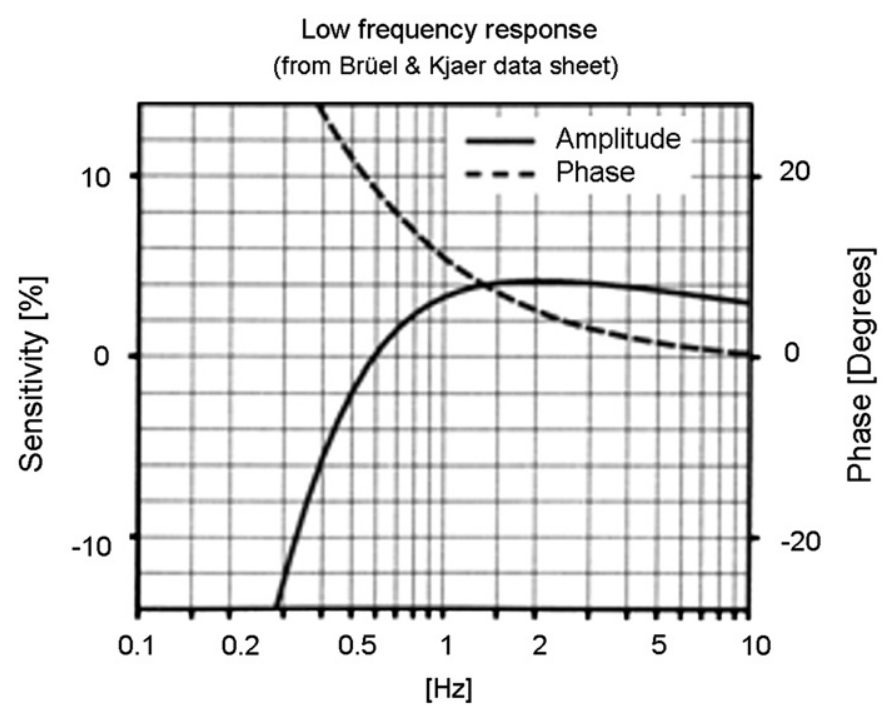

Fig. 4. Frequency response of the used accelerometers. Reference sensitivity: $995 \mathrm{mV} / \mathrm{g}$.

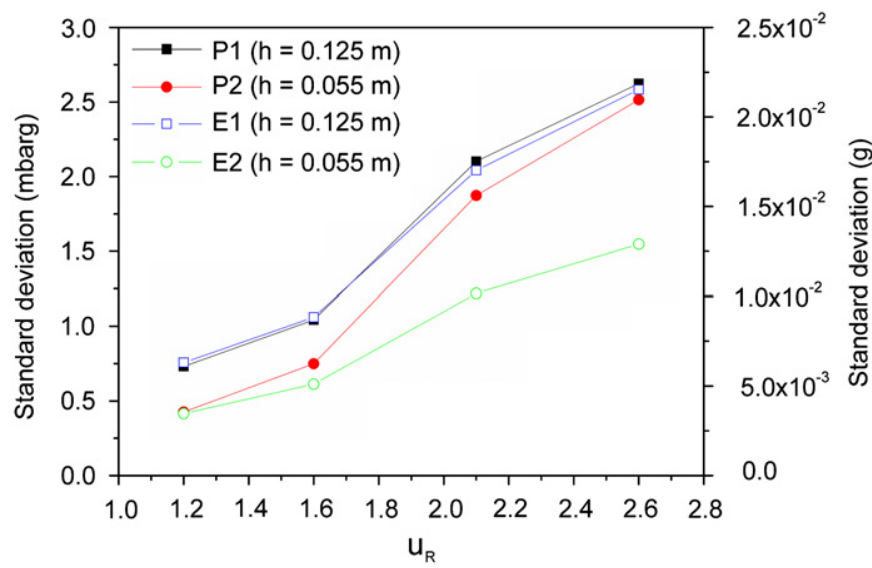

Fig. 5. Standard deviation of pressure and E signals vs. reduced air velocity.

According to that, in order to obtain the envelope from an experimental accelerometer signal, we need first an analytic function $Y(t)$. Consequently, though there are many ways of defining the imaginary part of a function, the Hilbert transform 
provides a unique way to obtain such an imaginary component. Therefore, the result of applying the Hilbert transform to an experimental accelerometer signal is an analytic function. Analytic signals have been broadly applied in vibration studies despite the fact that this technique might induce some errors in the calculation of the envelope that could influence its nonlinear characteristics (Harris and Piersol, 2002; Cheng et al., 2007).

\section{Experimental}

Accelerometry and pressure fluctuation signals were recorded in a cylindrical poly methyl metacrylate (PMMA) vessel of $0.07 \mathrm{~m}$ diameter and 1 meter high. The air distributor consists of 7 tuyeres that provide a homogeneous air distribution. Each tuyere has 8 holes $0.5 \mathrm{~mm}$ diameter giving a distributor total pressure drop between 290 and $750 \mathrm{~Pa}$ in the operating conditions. The a

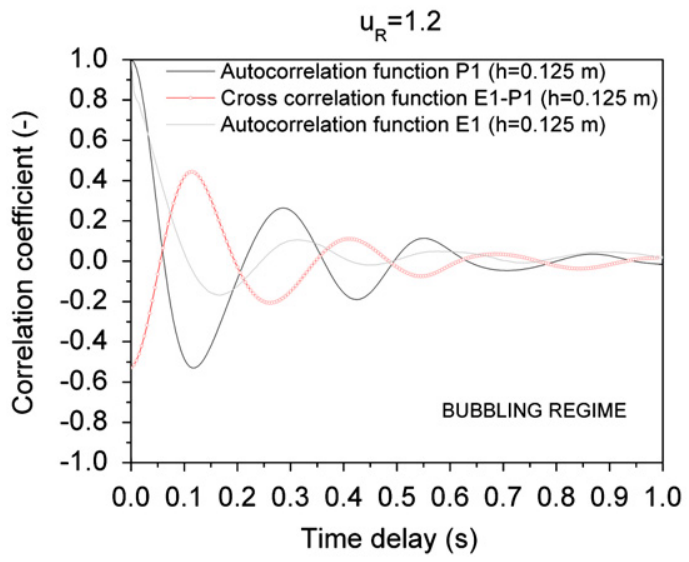

b

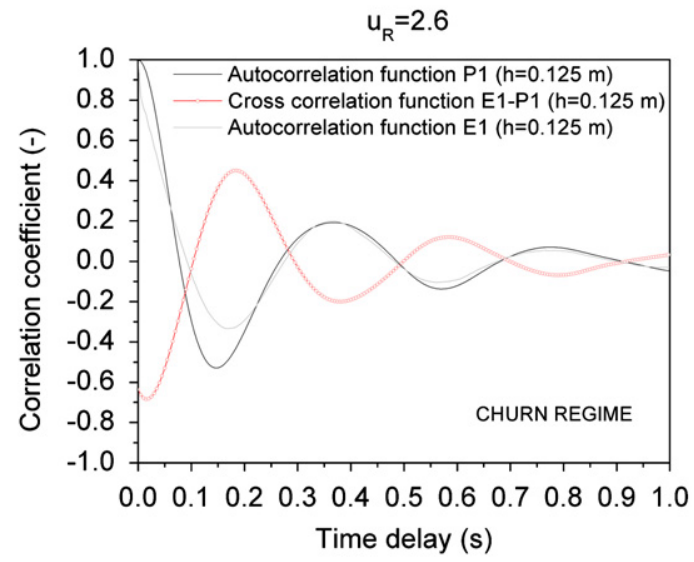

Fig. 6. Autocorrelation and cross-correlation functions for pressure and E signals: (a) $u_{\mathrm{R}} \quad 1.2$; (b) $u_{\mathrm{R}} \quad 2.6$.

a

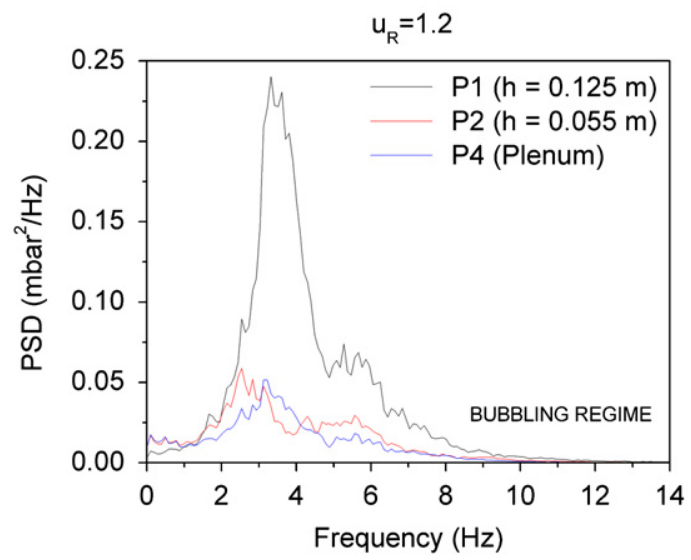

C

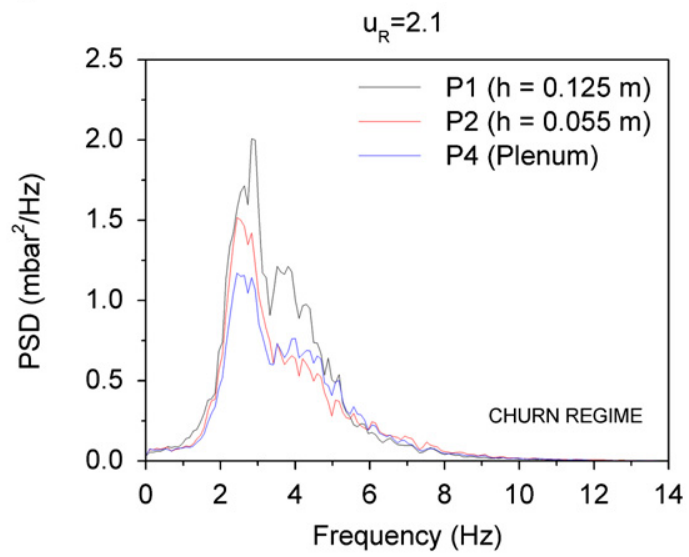

b

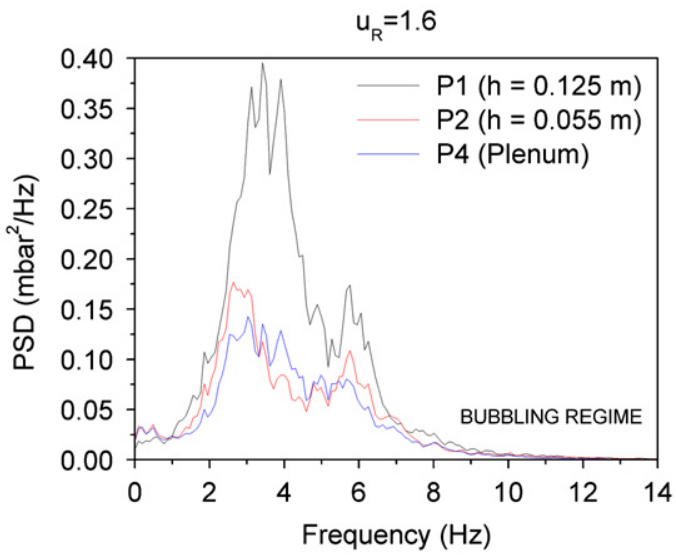

d

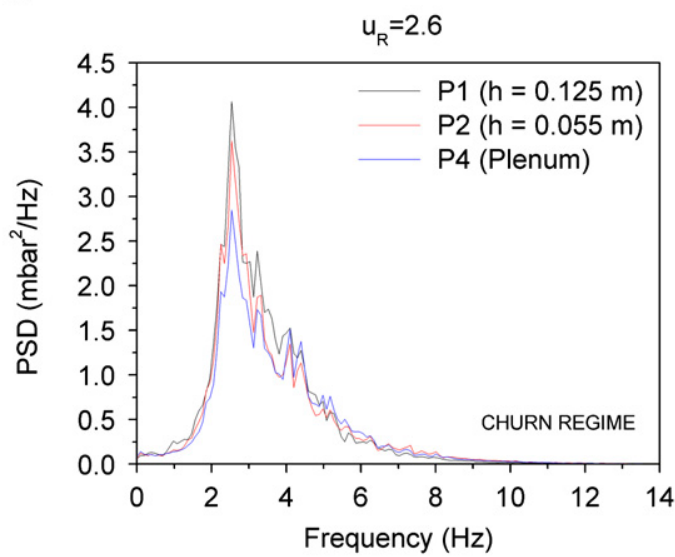

Fig. 7. Pressure spectra. $f_{\mathrm{s}} \quad 200 \mathrm{~Hz}$ : (a) $u_{\mathrm{R}}$ 1.2; bubbling regime (b) $u_{\mathrm{R}} \quad 1.6$, bubbling regime; (c) $u_{\mathrm{R}} \quad 2.1$, churn regime; (d) $u_{\mathrm{R}} \quad 2.6$, churn regime. 
a

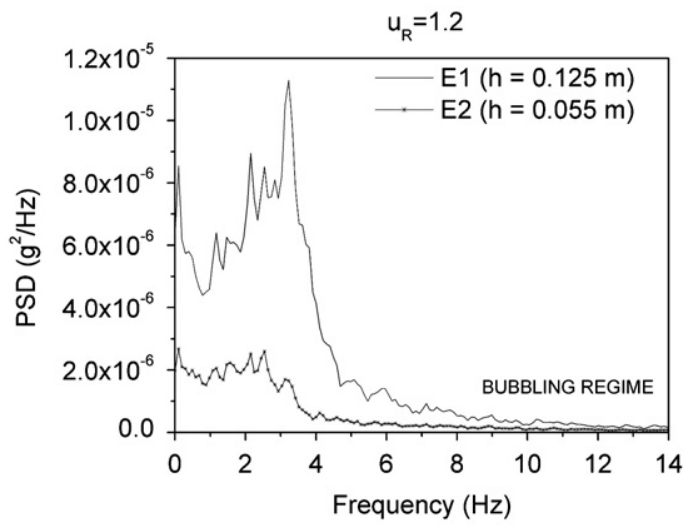

C

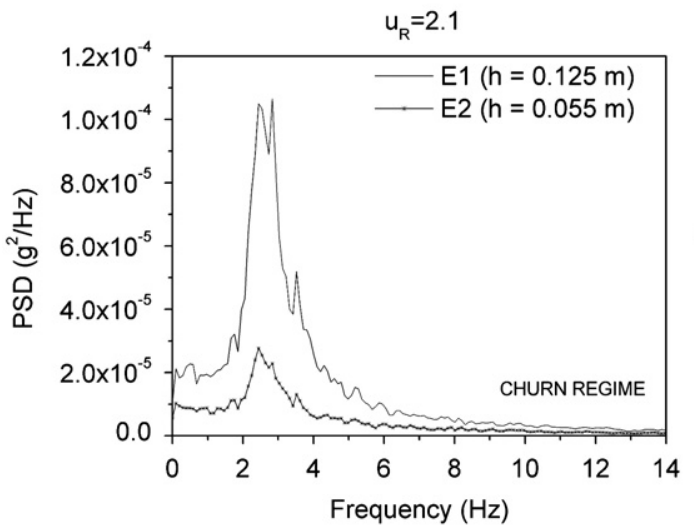

b

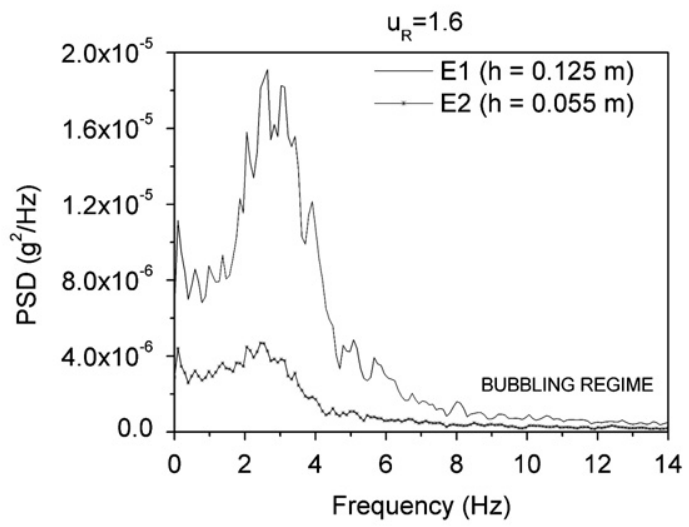

d

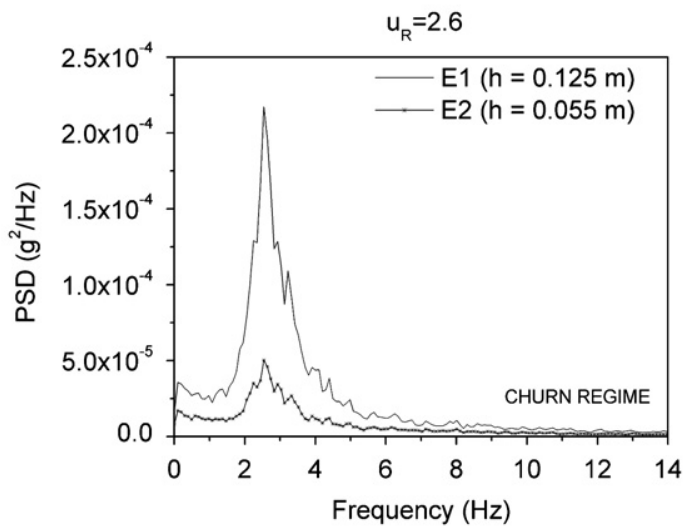

Fig. 8. E spectra. $f_{\mathrm{s}} 200 \mathrm{~Hz}$ : (a) $u_{\mathrm{R}} \quad 1.2$; bubbling regime (b) $u_{\mathrm{R}} \quad 1.6$, bubbling regime; (c) $u_{\mathrm{R}} \quad 2.1$, churn regime; (d) $u_{\mathrm{R}} \quad 2.6$, churn regime.

characteristics of the fluidized bed and particles are shown in Table 1.

Pressure and acceleration signals were measured during 5 min with a sampling frequency of, respectively, $200 \mathrm{~Hz}$ and $10 \mathrm{kHz}$ (to avoid resonant amplification in the accelerometers). Later, the envelope of the accelerometer signals was estimated with the Hilbert transform and subsequently resampled at $200 \mathrm{~Hz}$ in order to have the same size as the pressure signals. Simultaneously, pressure fluctuations were recorded with a frequency of $200 \mathrm{~Hz}$. Four runs at reduced air velocities $u_{0} / u_{\mathrm{mf}}$ of $1.2,1.6,2.1$ and 2.6 were performed to explore different operational conditions. The minimum fluidization high, $h_{\mathrm{mf}}$, was $0.165 \mathrm{~m}$. The pressure probes P1, P2 and P4 are located at 0.125 and $0.055 \mathrm{~m}$ from the distributor and inside the plenum, respectively. The acceler ometers E1 and E2 are placed at 0.125 and $0.055 \mathrm{~m}$ from the distributor, as close as possible to the pressure probes, respectively.

\subsection{Acceleration measurement system}

The horizontal vibrations of the vessel wall were collected by means of commercial accelerometers (Brüel and Kjaer Type 4507 $B$ 005) located in the external face of the wall by means of mounting slots supported with glue. The accelerometers are fitted to a NI 9233 (National Instruments) acquisition module inserted in a chassis NI cDAQ 9172 connected to a PC. The software used to acquire the signals is Labview 8.5. The sensors have a sensitivity of $995 \mathrm{mV} / \mathrm{g}$ and a resonant frequency of $16.3 \mathrm{kHz}$.

Special care was taken with the wire that connects each accelerometer with the data acquisition device to avoid triboelectric noise in the signal. The frequency response of the accelerometers is shown in Fig. 4.

\subsection{Pressure measurement system}

Pressure fluctuations were recorded by a probe $6 \mathrm{~cm}$ length and $4 \mathrm{~mm}$ i.d. connected to a pressure transducer PR3110 (Ellison Sensors) fitted to a NI 9205 data acquisition module. This module was also mounted in the chassis NI cDAQ 9172. The distance between the probe and the transducer is less than $0.2 \mathrm{~m}$. These sizes led to ensure that damping effects are negligible (van Ommen et al., 1999).

\section{Results}

\subsection{Information from visual observation}

The visual inspection of the fluidization state has revealed two types of fluidization regimes within the operating conditions explored: bubbling regime was observed for reduced air velocities $1.2 \leq u_{R} \leq 1.6$ and churn type regime was observed for $u_{R}>1.6$. The appearance of both states is qualitatively described below.

Bubbling regime: As described previously in literature, several bubbles simultaneously present within the bed that increase their size quickly with the distance from the distributor; as a result, the lower part of the vessel has a bubble distribution (bubble number and size) quite different from the upper part, Moreover, it has been observed that the bed geometry (narrow bed) strongly influences the resulting PSD, the narrow bed feature of the facility makes the natural bed frequency dominate the spectra. 
a

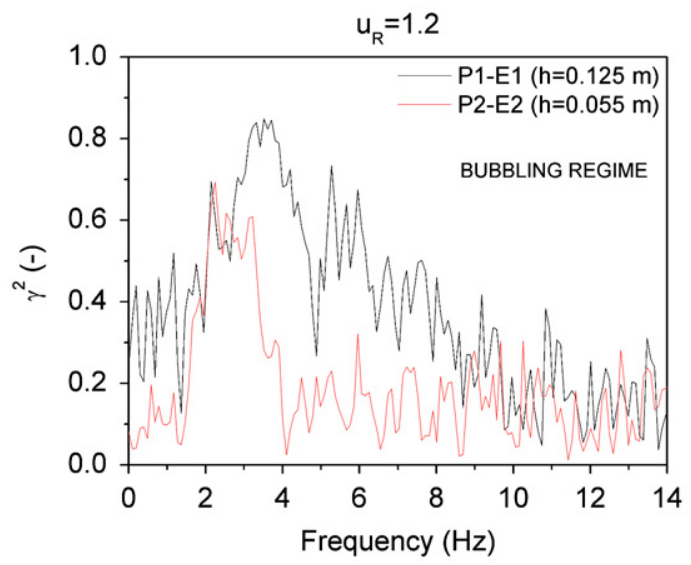

C

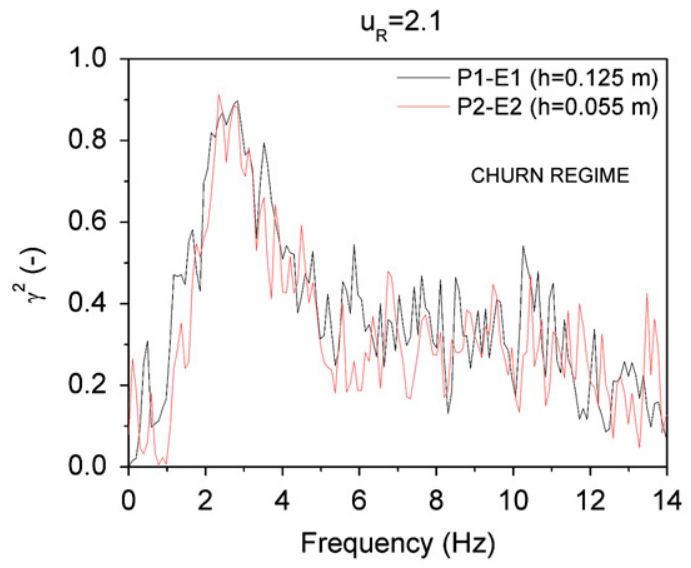

b

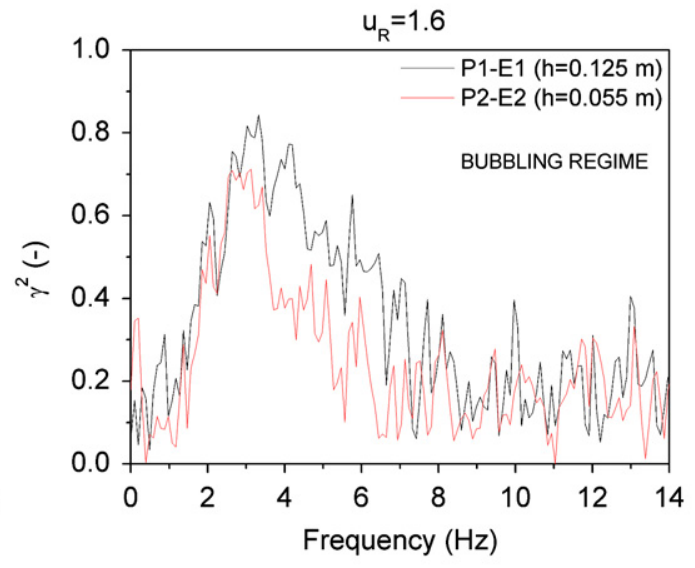

d

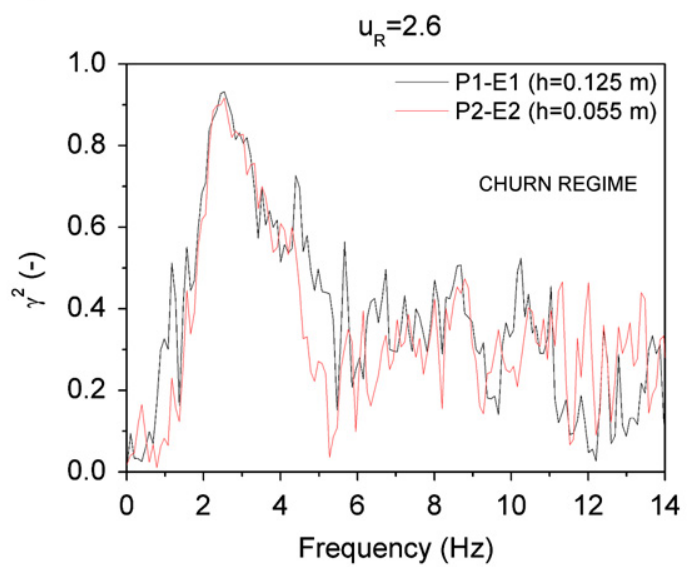

Fig. 9. Cross coherence coefficient. $f_{\mathrm{s}} \quad 200 \mathrm{~Hz}$ : (a) $u_{\mathrm{R}} \quad 1.2$; bubbling regime (b) $u_{\mathrm{R}} \quad 1.6$, bubbling regime; (c) $u_{\mathrm{R}} \quad 2.1$, churn regime; (d) $u_{\mathrm{R}} \quad 2.6$, churn regime.

Churn type regime: Controversy exists about the existence of this regime in gas solid FB (Hewitt and Jayanti, 1993). In this work, we refer to a form of developing slug flow in short pipes with the appearance of a churning motion. Consequently, that region of churn like flow is characterized by large bubbles with a diameter similar to the vessel size, which result in a vigorous fluidization process.

\subsection{Time domain comparison}

The time domain analysis is frequently used to gain knowledge of the temporal scale of the dynamical processes (fast or slow processes) underlying the time series under study. The probability structure of the time series is used to be characterized by means of its moments. Thus, the standard deviation $\sigma$ is a frequently used parameter for monitoring and diagnosis purposes of gas solid FB. In spite of the well known limitations of $\sigma$ (Johnsson et al., 2000), it can be used, for instance, either to detect the transition velocity, $u_{\mathrm{c}}$, towards turbulent fluidization or as an indicator to detect malfunctioning (van Ommen et al., 2004). The standard deviation of experimental pressure and E signals is shown in Fig. 5. It is observed that $\sigma$ behaves similarly for both the pressure and the accelerometer signals, increasing with the air velocity. The fact that the standard deviation presents the same trend for both signals points out the similarity between the amplitude information stored in both the accelerometer and the pressure signals. Consequently, it can be argued that the standard deviation estimated from accelerometer signals could replace the standard deviation of the pressure signal in some reported applications such as to determine malfunctioning and for estimating the minimum fluidization velocity.

Since the probability structure of a time series is usually described in terms of second order statistics, the correlation function is frequently used as a measurement of the temporal structure of the time series. It is well known that the autocorrela tion function assumes a linear relationship between the data. Nevertheless, it provides useful information about dynamical correlations existing within time series. Thus, the autocorrelation and the cross correlation functions for P1 and E1 signals estimated for $u_{R}=1.2$ and $u_{R}=2.6$, are shown in Fig. 6 . The results from the autocorrelation analysis reveal that both P1 and E1 measurements exhibit a similar temporal structure. Moreover, the computed cross correlation function, between P1 and E1 time series, shows clearly that both phenomena, the pressure fluctuations and the mechanical vibrations, are related.

\subsection{Frequency domain analysis}

In order to study the characteristic frequencies of all the measured signals, let us focus the attention on studying the spectral properties of the correlation functions. Thus, as stated above, Welch's method is used for the PSD estimation and the coherence function, $\gamma^{2}$, is subsequently computed to quantify the degree of linear coupling between pressure and $\mathrm{E}$ signals at a given frequency. According to that, from the characteristic frequencies of the measured pressure fluctuation signals shown in Fig. 7, it can be observed that the energy of the spectra is distributed mainly below $10 \mathrm{~Hz}$ with a dominant frequency at 
a

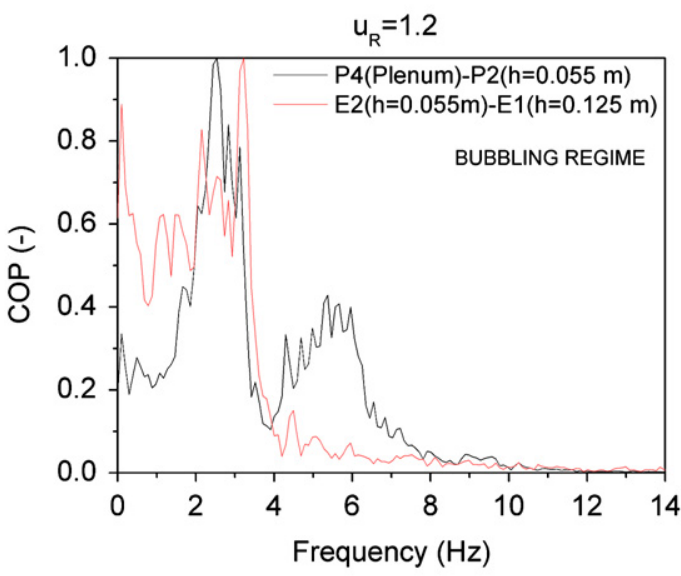

C

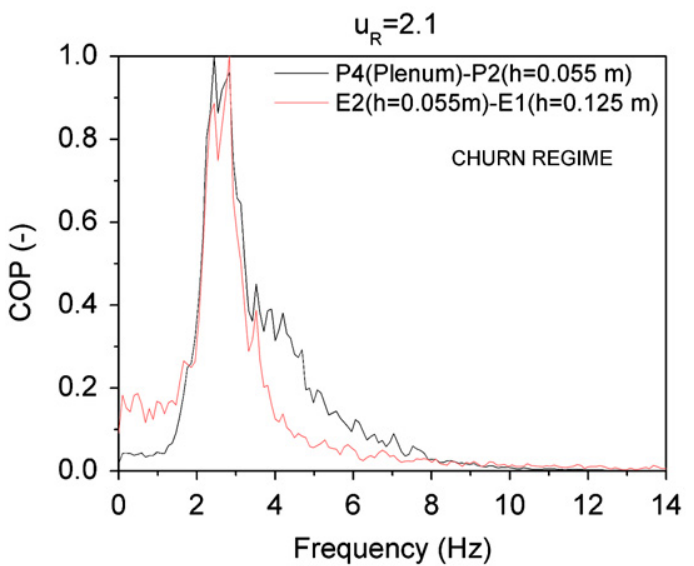

b

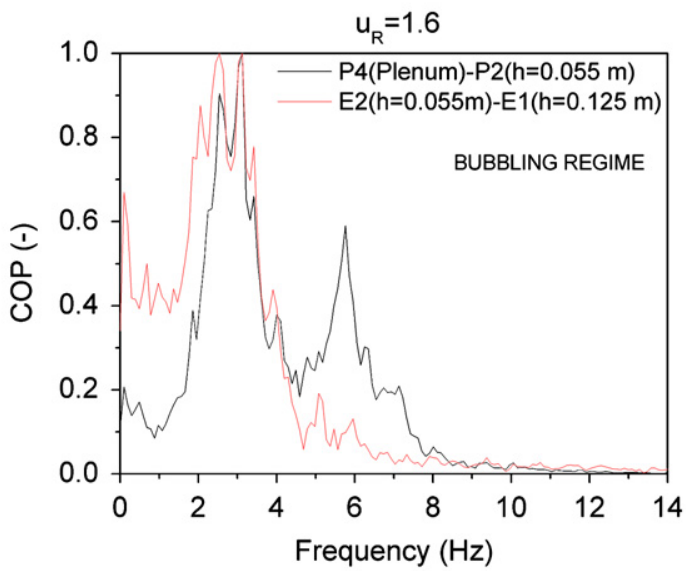

d

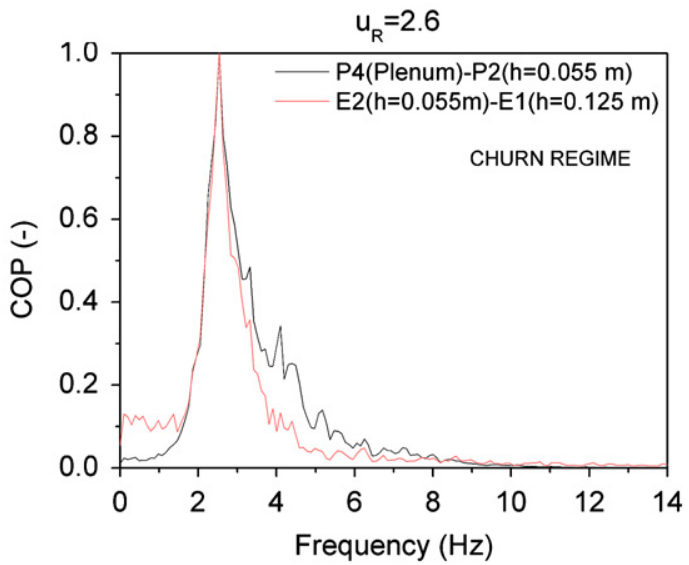

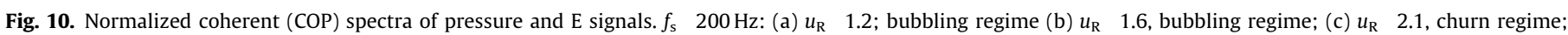
(d) $u_{R} \quad 2.6$, churn regime.

$2.5 \mathrm{~Hz}$, which correspond to the natural bed frequency $\left(f_{\text {bulk }}=2.5 \mathrm{~Hz}\right.$ (Baskakov et al., 1986), for all operational conditions covered. Moreover, the dynamical features of the two flow conditions qualitatively described previously, from visual observation, are now explained by the spectra shown on Fig. 7. Thus, during the bubbling regime, it is observed how the different bubble distribution along the bed give rise to pressure spectra whose energy is quite dependent on the probe position (Fig. 7a,b). In contrast, during the churn like behaviour (Fig. 7c,d) the promoted vigorous fluidization equalizes the energy of the spectra estimated from the different position.

The E signals shown in Fig. 8 provide a spectra characterized by an energy content distributed below $10 \mathrm{~Hz}$ as was observed for the pressure fluctuation signal. Moreover, the dominant frequency at $2.5 \mathrm{~Hz}$ is also distinguishable and, the different dynamical features characterizing the observed bubbling and churn like flow are clearly reflected on the envelope spectra. However, in contrast to pressure signals, which during the churn like behaviour (Fig. 7c,d) equalize the energy of the spectra estimated from the different position, the accelerometer power seems not to be influenced for that churning flow, in the sense that the energy relation between signals taken at different position remains unchanged. Nevertheless, as shown by the previous spectra (Figs. 7 and 8) there is a clear relation between the measured pressure and E signals. Accordingly, Fig. 9 shows the coherence function between pressure and E signals, which were collected simultaneously at the same bed height. As expected, a high coherence is observed for frequencies ranging between 2 and $4 \mathrm{~Hz}$, especially for measurements collected at P1 and E1 positions, with a coherence function, $\gamma^{2}>0.8$, which reveals the existence of a high degree of coupling between the processes occurring at that frequency interval. This is in agreement with the previous results reached in the time series analysis, concluding that both phenomena, the pressure fluctuations and the mechanical vibrations, are related.

\subsection{COP/IOP analysis}

It has been reported (van der Schaaf et al., 2002) that some dynamic phenomena such as bubble coalescence, gas flow fluctuation, bubble eruption and bed mass oscillation generate fast pressure waves that propagate in such a way that they can be measured almost instantaneously through the entire bed. Conse quently, pressure measurements taken at different bed positions will show coherence due to those fast travelling waves. In contrast, other phenomena such as gas bubbles and turbulence only generate a local fluctuation in pressure corresponding to low coherence phenomena. Thus, in order to distinguish the different components in the E signals, the normalized coherent (COP) and incoherent (IOP) output power were calculated for the pressure signals collected at the plenum and at some in bed positions, and for accelerometry signals taken at two different positions within the bed (Figs. 10 and 11). 
a

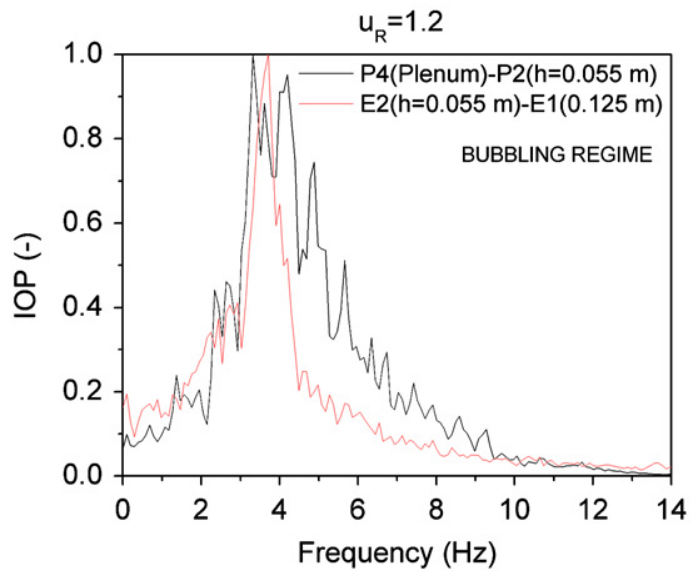

C

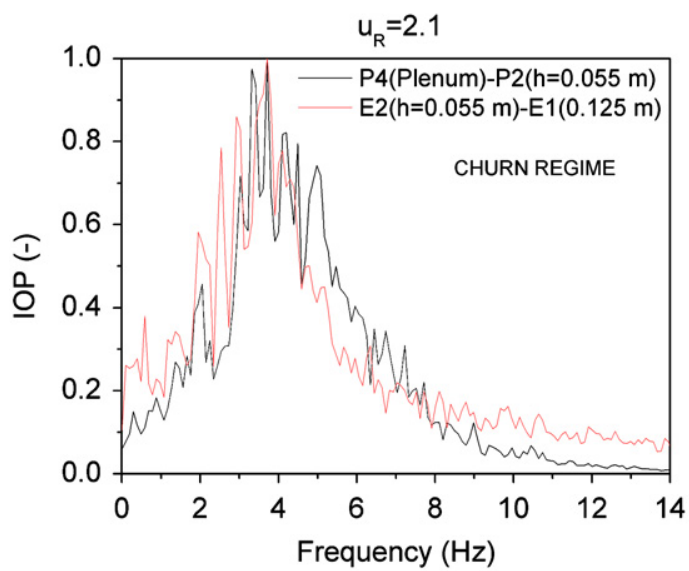

b

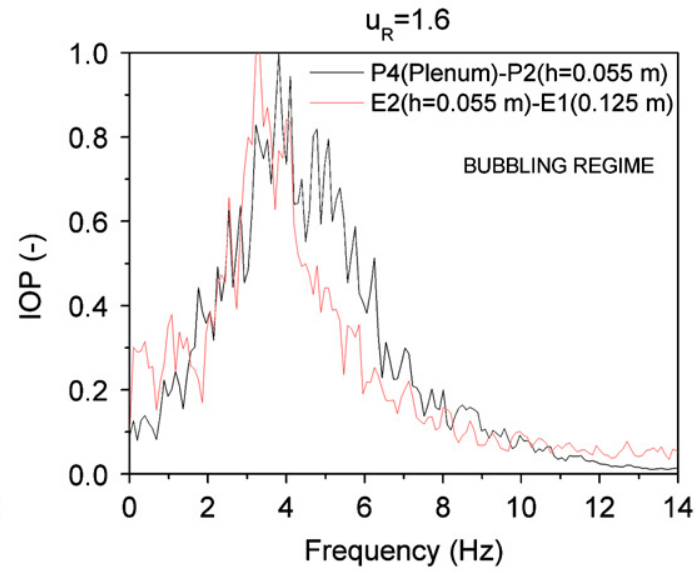

d

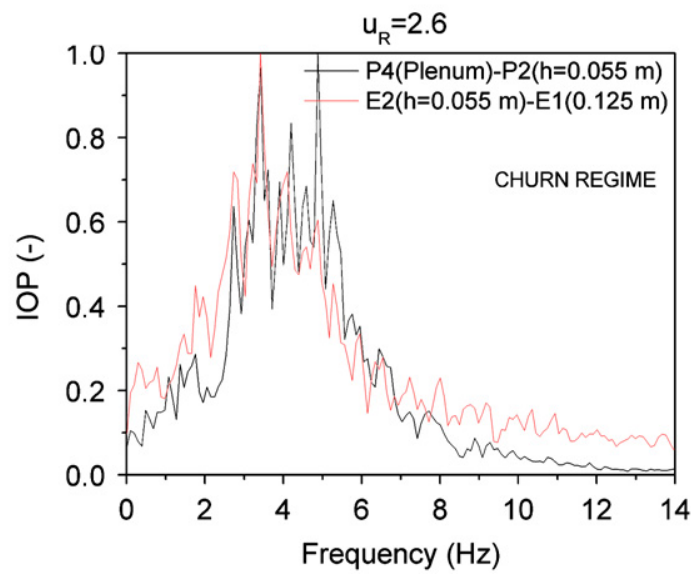

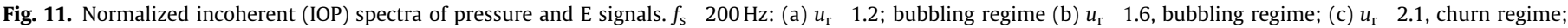
(d) $u_{\mathrm{r}}$ 2.6, churn regime.

The resulting normalized COP function of pressure and E signals are shown in Fig. 10, where the contribution due to the bed mass oscillation, $f_{\text {bulk }}=2.5 \mathrm{~Hz}$ (Baskakov et al., 1986) is clearly distinguishable on the COP spectra for both pressure and E time series. Moreover, it is observed that for bubble regime, the second maximum detected by the COP estimated from the pressure fluctuation signals does not appear on the COP computed from E signals that shows a low coherence for the frequencies above $4 \mathrm{~Hz}$. That fact might be attributed to either vessel response or wave propagation mechanism. In contrast the COP for both the pressure and the E signal for churn like flow exhibit similar characteristics.

With regard to the normalized IOP analysis (Fig. 11), the high similarity between the pressure and the E spectra is remarkable, which points out that the local information due to gas bubble and turbulence is barely affected by the measurement technique. Consequently, from a frequency domain point of view there is no difference in using E or pressure signal to study local phenomena.

\section{Conclusions}

The low frequency information encoded within an acceler ometer signal measured by low cost commercial accelerometers provides useful information on bulk and bubble dynamics that can be used for gas solid fluidized bed monitoring, and dynamic diagnosis purposes.
The proposed methodology, consisting on extracting the low frequency information carried within an accelerometer signal by means of the Hilbert transform method, succeeds in recovering the dynamical information below $20 \mathrm{~Hz}$, which has been shown to be directly related to the conventional fluidized bed pressure fluctuation measurements.

The time domain analysis, in terms of standard deviation and autocorrelation function, reveals that both the pressure fluctua tion and the mechanical vibration have a similar probability structure. Furthermore, from the cross correlation analysis be tween the pressure and E signals, it is concluded that they are related processes.

The frequency domain analysis confirms the previous results drawn from the time series analysis, pointing out the existing relation between pressure fluctuation and vibration phenomena. Thus, the PSD of both pressure and E signals reflect the different dynamical features characterizing the observed bubbling and churning like flow at the operational condition covered. Moreover, the coherence function computed between pressure and E signal exhibits values approaching unity for frequencies ranging between 2 and $4 \mathrm{~Hz}$.

Finally, the COP/IOP analysis of the E signals exhibits similar characteristics as the COP/IOP analysis of pressure fluctuation signals, opening the possibility of using low frequency accel erometer instead of conventional pressure transducer for mon itoring and dynamic diagnosis purposes. However, despite the fact that the results at lab scale level are very promising, more 
research on low frequency accelerometry is needed before replacing pressure measurements for the monitoring of industrial gas solid fluidized beds. Other aspects such as insulation thick ness and vessel material can influence the quality and quantity of the accelerometer data. Besides, multi resolution and state space analysis should be complementarily applied with frequency and time domain analysis to get further knowledge on the origin and characteristics of the E signals.

\section{Notation}

\section{Uppercase letters}

$A(t)$ instant amplitude of the signal

$N_{\mathrm{s}} \quad$ number of averaged periodograms

$Y(t)$ analytic signal

\section{Lowercase letters}

$a(t)$ real part of the Hilbert transform

$b(t)$ imaginary part of the Hilbert transform

$d_{\mathrm{p}} \quad$ mean particle size, $\mathrm{m}$

$f$ frequency, $\mathrm{Hz}$

$f_{\text {bulk }}$ frequency of the bulk oscillations, $\mathrm{Hz}$

$f_{\mathrm{s}}$ sample frequency, $\mathrm{Hz}$

$h_{\mathrm{mf}}$ minimum fluidization high, $\mathrm{m}$

$u_{0} \quad$ air velocity, $\mathrm{m} / \mathrm{s}$

$u_{\mathrm{mf}}$ minimum fluidization velocity, $\mathrm{m} / \mathrm{s}$

$u_{\mathrm{R}} \quad$ reduced velocity, $u_{0} / u_{\mathrm{mf}}$

\section{Greek letters}

$$
\begin{array}{ll}
\varepsilon_{\mathrm{mf}} & \text { minimum fluidization voidage } \\
\sigma & \text { amplitude, mbarg, } \mathrm{g} \\
\Phi & \text { Sphericity } \\
\rho_{\mathrm{mf}} & \text { minimum fluidization density, } \mathrm{kg} / \mathrm{m}^{3} \\
\rho_{\mathrm{p}} & \text { particle density, } \mathrm{kg} / \mathrm{m}^{3}
\end{array}
$$

\section{Abbreviations}

COP coherent part of the power spectrum, $\operatorname{mbarg}^{2} / \mathrm{Hz}, \mathrm{g}^{2} / \mathrm{Hz}$ IOP incoherent part of the power spectrum, $\operatorname{mbarg}^{2} / \mathrm{Hz}, \mathrm{g}^{2} / \mathrm{Hz}$ PSD power spectral density, mbarg $^{2} / \mathrm{Hz}, \mathrm{g}^{2} / \mathrm{Hz}$

\section{Acknowledgements}

The authors would like to thank the financial support from the Spanish Ministry of Research, project CTQ2006 15525 C02 01.

\section{References}

Abbasi, M., Sotudeh-Gharebagh, R., Mostoufi, N., Mahjoob, M.J., 2009. Nonintrusive monitoring of bubbles in a gas-solid fluidized bed using vibration signature analysis. Powder Technology 196, 278-285.

Amos, G., Rhodes, M.J., Benkreira, H., 1996. Calculation of optic fibres calibration curves for the measurement of solids volume fractions in multiphase flows. Powder Technology 88, 107-121.

Baskakov, A.P., Tuponogov, V.P., Filipovsky, N.F., 1986. A study of pressure fluctuations in a bubbling fluidized bed. Powder Technology 45, 113-117.

Bi, H.T., 2007. A critical review of the complex pressure fluctuation phenomenon in gas-solids fluidized beds. Chemical Engineering Science 62, 3473-3493.

Bi, H.T., Grace, J.R., Zhu, J., 1995. Propagation of pressure waves and forced oscillations in gas-solid fluidized beds and their influence on diagnosis of local hydrodynamics. Powder Technology 82, 239-253.

Boyd, J.W.R., Varley, J., 2001. The uses of passive measurement of acoustic emissions from chemical engineering processes. Chemical Engineering Science 56, 1749-1767.

Boyd, J.W.R., Varley, J., 2002. Measurement of gas solid hold-up in bubble columns from low frequency acoustic emissions. Chemical Engineering Journal 88, $111-118$.
Brereton, C.M.H., Grace, J.R., 1993. Microstructural aspects of the behaviour of circulating fluidized beds. Chemical Engineering Science 48, 2565-2572.

Briongos, J.V., Aragón, J.M., Palancar, M.C., 2006a. Phase space structure and multiresolution analysis of gas solid fluidized beds hydrodynamics. Part I: The EMD approach. Chemical Engineering Science 61, 6963-6980.

Briongos, J.V., Aragón, J.M., Palancar, M.C., 2006b. Fluidised bed dynamics diagnosis from measurements of low-frequency out-bed passive acoustic emissions. Powder Technology 162, 145-156.

Briongos, J.V., Aragón, J.M., Palancar, M.C., 2007. Phase space structure and multiresolution analysis of gas solid fluidized bed hydrodynamics. Part II: Dynamic Analysis. Chemical Engineering Science 62, 2865-2879.

Briongos, J.V., Guardiola, J., 2003. Free top fluidized bed surface as a source of hydrodynamic data. Powder Technology 134, 133-144.

Brown, R.C., Brue, E., 2001. Resolving dynamical features of fluidized beds from pressure fluctuations. Powder Technology 119, 68-80.

Cents, A.H.G., Brilman, D.W.F., Versteeg, G.F., Wijnstra, P.J., Regtien, P.P.L., 2004. Measuring bubble, drop and particle sizes in multiphase systems with ultrasound. AIChE Journal 50, 2750-2762.

Cheng, J., Yu, D., Yu, Y., 2007. The application of energy operator demodulation approach based on EMD in machinery fault diagnosis. Mechanical Systems and Signal Processing 21, 668-677.

Cody, G.D., Goldfarb, D.J., Storch, G.V., Norris, A.N., 1996. Particle granular temperature in gas fluidized beds. Powder Technology 87, 211-232.

Daw, C.S., Lawkins, W.F., Downing, D.J., Clapp Jr., N.E., 1990. Chaotic characteristics of a complex gas-solids flow. Physical Review A 41, 1179-1181.

Finney, C.E.A., Daw, C.S., Halow, J.S., 1998. Measuring slugging bed dynamics with acoustic sensors. KONA: Powder and Particle 16, 125-135.

Gatti, P.L., Ferrari, V., 1999. Applied Structural and Mechanical Vibrations: Theory, Methods and Measuring Instrumentation, 1st ed Taylor \& Francis, London.

Harris, C.M., Piersol, A.G., 2002. Harris' Shock and Vibration Handbook, 5th ed McGraw Hill.

Herrera, C.A., Levy, E.K., Ochs, J., 2002. Characteristics of acoustic standing waves in fluidized beds. AIChE Journal 48, 503-513.

Hewitt, G.F., Jayanti, S., 1993. To churn or not to churn. International Journal of Multiphase Flow 19, 527-529.

Huang, N.E., Wu, Z., Long, S.R., Arnold, K.C., Chen, X., Blank, K., 2009. On instantaneous frequency. Advances in Adaptive Data Analysis 1, 177-229.

Johnsson, F., Zijerveld, R.C., Schouten, J.C., van den Bleek, C.M., Leckner, B., 2000. Characterization of fluidization regimes by time-series analysis of pressure fluctuations. International Journal of Multiphase Flow 26, 663-715.

Kage, H., Iwasaki, N., Yamaguchi, H., Matsuno, Y., 1991. Frequency analysis of pressure fluctuation in fluidized bed plenum. Journal of Chemical Engineering of Japan 24, 76-81.

Li, J., Kwauk, M., 2001. Multiscale nature of complex fluid-particle systems. Industrial \& Engineering Chemistry Research 40, 4227-4237.

$\mathrm{Lu}, \mathrm{X}$., Li, H., 1999. Wavelet analysis of pressure fluctuation signals in a bubbling fluidized bed. Chemical Engineering Science 75, 113-119.

Louge, M., Opie, M., 1990. Measurements of the effective dielectric permittivity of suspensions. Powder Technology 62, 85-94.

Nigam, N.C., 1983. Introduction to Random Vibrations. MIT Press. ISBN: 0-262$14035-7$.

Norton, M.P., 1989. Fundamentals of Noise and Vibration Analysis for Engineers. Cambridge University Press. ISBN: 0-521-34148-5.

Sasic, S., Leckner, B., Johnsson, F., 2007. Characterization of fluid dynamics of fluidized beds by analysis of pressure fluctuations. Progress in Energy and Combustion Science 33, 453-496.

Satija, S., Fan, L.S., 1985. Characteristics of slugging regime and transition to turbulent regime for fluidized beds of large particles. AIChE Journal 31, 1554-1562.

Schouten, J.C., van den Bleek, C.M., 1992. Chaotic hydrodynamics of fluidization: consequences for scaling and modelling of fluid bed reactors. AIChE Symposium Series 88, 70-84.

Solimene, R., Marzocchella, A., Ragucci, R., Salatino, P., 2007. Laser diagnostics of hydrodynamics and gas-mixing induced by bubble bursting at the surface of gas-fluidized beds. Chemical Engineering Science 62, 94-108.

Sun, J.G., Chen, M.M., Chao, B.T., 1994. Modeling of solids global fluctuations in bubbling fluidized beds by standing surface waves. International Journal of Multiphase Flow 20, 315-338.

Tsujimoto, H., Yokoyama, T., Huang, C.C., Sekiguchi, I., 2000. Monitoring particle fluidization in a fluidized bed granulator with an acoustic emission sensor. Powder Technology 113, 88-96.

Van der Schaaf, J., Schouten, J.C., Johnsson, F., van den Bleek, C.M., 2002. Nonintrusive determination of bubble and slug length scales in fluidized beds by decomposition of the power spectral density of pressure time series. International Journal of Multiphase Flow 28, 865-880.

Van Ommen, J.R., Coppens, M.O., van den Bleek, C.M., Schouten, J.C., 2000. Early warning of agglomeration in fluidized beds by attractor comparison. AIChE Journal 46, 2183-2197.

Van Ommen, J.R., de Korte, R.J., van den Bleek, C.M., 2004. Rapid detection of defluidization using the standard deviation of pressure fluctuations. Chemical Engineering and Processing 43, 1329-1335.

Van Ommen, J.R., Schouten, J.C., van der Stappen, M.L.M., van den Bleek, C.M., 1999. Response characteristics of probe-transducer systems for pressure measurements in gas-solid fluidized beds: how to prevent pitfalls in dynamic pressure measurements. Powder Technology 106, 199-218. 
Werther, J., 1999. Measurement techniques in fluidized beds. Powder Technology $102,15-36$.

Yates, J.G., Simons, S.J.R., 1994. Experimental methods in fluidization research. International Journal of Multiphase Flow 20, 297-330.

Zhang, H., Johnston, P.M., Zhu, J.X., de Lasa, H.I., Bergougnou, M.A., 1998. A novel calibration procedure for a fiber optic solids concentration probe. Powder Technology 100, 260-272.
Zhao, G.-B., Yang, Y.-R., 2003. Multiscale resolution of fluidized-bed pressure fluctuations. AIChE Journal 49, 869-882.

Zijerveld, R.C., Johnsson, F., Marzocchella, A., Schouten, J.C., van den Bleek, C.M. 1998. Fluidization regimes and transitions from fixed bed to dilute transport flow. Powder Technology 95, 185-204.

Zukowski, W., 2001. An acoustic method of studying sequential explosions during gas combustion in bubbling fluidized beds. Combustion and Flame 125, 1075-1082. 